\title{
Specification of progression in glaucomatous visual field loss, applying locally condensed stimulus arrangements
}

\author{
Jukka Nevalainen • Jens Paetzold • Eleni Papageorgiou • Pamela A. Sample • \\ John P. Pascual • Elke Krapp • Bettina Selig • Reinhard Vonthein • Ulrich Schiefer
}

Received: 8 November 2008 / Accepted: 15 June 2009/Published online: 29 July 2009

(C) The Author(s) 2009. This article is published with open access at Springerlink.com

\begin{abstract}
Purpose The goal of this work was to (i) determine patterns of progression in glaucomatous visual field loss, (ii) compare the detection rate of progression between locally condensed stimulus arrangements and conventional $6^{\circ} \times 6^{\circ}$ grid, and (iii) assess the individual frequency distribution of test locations exhibiting a local event (i.e., an abrupt local deterioration of differential luminance sensitivity (DLS) by more than $-10 \mathrm{~dB}$ between any two examinations).

Methods The visual function of 41 glaucomatous eyes of 41 patients (16 females, 25 males, 37 to 75 years old) was examined with automated static perimetry (Tuebingen Computer Campimeter or Octopus 101-Perimeter). Stimuli were added to locally enhance the spatial resolution in suspicious regions of the visual field. The minimum follow-up was four subsequent sessions with a minimum of 2-month (median 6-month) intervals between each session. Progression was identified using a modified pointwise linear regression (PLR)
\end{abstract}

J. Nevalainen

Department of Ophthalmology, University of Oulu,

Oulu, Finland

J. Nevalainen · J. Paetzold · E. Papageorgiou • E. Krapp •

B. Selig $\cdot$ U. Schiefer $(\bowtie)$

Centre for Ophthalmology/Institute for Ophthalmic Research,

University of Tübingen,

Schleichstr. 12-16,

72076 Tübingen, Germany

e-mail: ulrich.schiefer@med.uni-tuebingen.de

P. A. Sample · J. P. Pascual

Hamilton Glaucoma Center, University of California,

San Diego, USA

\section{R. Vonthein}

Institute of Medical Biometry and Statistics,

University of Lübeck,

Lübeck, Germany method and a modified Katz criterion. The presence of events was assessed in all progressive visual fields.

Results Eleven eyes (27\%) showed progression over the study period (median 2.5 years, range $1.3-8.6$ years). Six (55\%) of these had combined progression in depth and size and five eyes (45\%) progressed in depth only. Progression in size conformed always to the nerve fiber course. Seven out of $11(64 \%)$ of the progressive scotomata detected by spatially condensed grids would have been missed by the conventional $6^{\circ} \times 6^{\circ}$ grid. At least one event occurred in $64 \%$ of all progressive eyes. Five of 11 (46\%) progressive eyes showed a cluster of events.

Conclusions The most common pattern of progression in glaucomatous visual fields is combined progression in depth and size of an existing scotoma. Applying individually condensed test grids remarkably enhances the detection rate of glaucomatous visual field deterioration (at the expense of an increased examination time) compared to conventional stimulus arrangements.

Keywords Glaucoma · Progression · Perimetry · Condensation $\cdot$ Event

\section{Introduction}

Glaucomatous visual field progression algorithms assess changes in local differential luminance sensitivity (DLS) across time. The appearance of new scotomata or changes to existing field defects can be monitored across time using either trend or event analysis. So far, there is no generally accepted definition for progression of glaucomatous visual field loss. Trend analyses fit linear regression to DLS values and compare the obtained slope to the distribution of slopes from hypothetically non-progressing thresholds. 
Event analyses compare thresholds in prior exams to those in subsequent exams and compare the observed threshold changes to empiric distributions of threshold changes from stable glaucoma patients. Both of these approaches are reasonable since according to earlier studies the most common pattern of progression in glaucoma is deepening of an existing scotoma $[4,25,26]$, yet the field has not converged upon a single "gold standard" for visual field progression.

Due to practical considerations of time in the clinic, the spatial resolution for the detection of glaucomatous changes in standard automated perimetry has historically been restricted to a $6^{\circ} \times 6^{\circ}$ rectangular grid. However, spatially denser grids can detect glaucomatous defects earlier than conventional $6^{\circ} \times 6^{\circ}$ test point arrangements $[1,30,36,39]$. Local condensation in functionally or morphologically suspicious areas can enhance spatial resolution without unreasonably extending the examination duration [30]. The test grid is condensed within a "region of interest" (ROI) of the visual field as circumscribed by the clinician. An ROI is chosen for each patient by consulting neighborhoods of visual field areas adjacent to their existing scotoma (Scotoma-Oriented Perimetry, SCOPE) or to the visual field areas associated with morphologically suspicious areas as determined by optic disc and nerve fiber layer photographs (Fundus-Oriented Perimetry $=$ FOP). In addition to improving the detection of glaucomatous visual field loss, local condensation of stimuli could be a useful approach for monitoring disease progression.

To our knowledge, this is the first study to analyze the patterns of visual field defect (VFD) progression in glaucoma with locally condensed stimulus arrangements. The aims of this study are to (i) determine the patterns of progression in glaucomatous visual field defects, (ii) compare the detection rate of VFD progression of locally condensed grids with that of a conventional $6^{\circ} \times 6^{\circ}$ grid and (iii) assess the individual frequency of test locations exhibiting a local event.

\section{Materials and methods}

\section{Patients}

Progression in conventional and condensed test grids was analyzed retrospectively in 41 eyes of 41 patients (16 females, 25 males) from the Tuebingen University Eye Hospital with adequately treated glaucoma stage 1 or higher according to Mills classification [27]: 34 had primary openangle glaucoma, four had low-tension glaucoma, one had pigmentary glaucoma and two glaucomas could not be classified. Ages ranged between 37 and 75 years (median 60 years). All patients had $|<8|$ Dsph; $|<3|$ Dcyl; best refracted central visual acuity $>10 / 20$; no relevant opacities of the central refractive media; no history or signs of other optic neuropathies or other diseases affecting the visual field than glaucoma, and were not receiving miotic drugs. Patients with advanced optic disc cupping that exceeded two clock hours were excluded from this study to avoid a floor effect, i.e., further progression may not be detectable in these areas due to end-stage visual field loss. Furthermore, patients with abnormalities of the anterior chamber as determined by gonioscopy (Goldmann 3 mirror contact lens), or at risk of an angle closure were excluded from this study. Fundus morphology was assessed with direct ophthalmoscope using red-free illumination and indirect fundus lens $(78 \mathrm{D}$ or $90 \mathrm{D})$ at the slit lamp. The protocol adhered to the tenets of the Declaration of Helsinki.

All patients had a manifest glaucoma according to the European Glaucoma Society (EGS) criterion [9] with unequivocal glaucomatous alterations of optic nerve head morphology and/or retinal nerve fiber layer (RNFL), and manifest visual field defects. Glaucomatous optic nerve head morphology was characterized using stereophotographs, with glaucomatous alterations defined by diffuse thinning or focal notching of the neuroretinal rim, deviation from the ISNT rule [19], vertical cup/disc ratio exceeding 0.6 , optic disc hemorrhage[s], or bared circumlinear vessels. Glaucomatous RNFL defects were defined as wedge-shaped, circumscribed, or focal losses.

\section{Visual fields}

Examinations were conducted with threshold-estimating static, standard automated perimetry (full-threshold 4-2-1 strategy, with at least three reversals) in a minimum of four subsequent sessions (one baseline session and three follow-up sessions). All 41 eyes were initially examined with the Tuebingen Computer Campimeter (TCC, manufactured at the Tuebingen University Eye Hospital). Thirty-two eyes finished the sessions before the TCC was replaced with the Octopus 101. The remaining nine eyes from autumn 2003 onward were tested with the Octopus 101 perimeter (Haag-Streit Inc., Koeniz, Switzerland). For both perimeters, a grid similar to the Humphrey pattern 30-2, with DLS values obtained in 72 locations spaced according to a $6^{\circ} \times 6^{\circ}$ grid was used.

The TCC used a video display unit as opposed to the cupola used by the Octopus 101. The Tuebingen group had previously determined age-related normative values for both the TCC (about 1996) and Octopus 101 perimeter as an order of the manufacturer Haag-Streit. A subgroup was tested on both devices, and since the difference of DLS at all test points was less than $1 \mathrm{~dB}$, transformation of the normative values of the Octopus 101 instrument was unnecessary for comparison to the TCC [17].

In all visual field examinations, additional test points were inserted between the original "coarse" stimulus 
arrangements. Regions of interest (ROI) were defined by the examiner to locally enhance spatial resolution in visual field areas related to anatomically suspicious regions like notches, hemorrhages, and circumscribed RFNL defects or related functionally to circumscribed visual field defects (Fig. 1). ROIs were defined as the area surrounding at least three visual field test locations with $p$-values $<0.05$ (according to the TD plot) in the relating upper or lower hemifield taking the course of the nerve fibers into consideration. Normal visual field test locations of the original $6^{\circ} \times 6^{\circ}$ grid, adjacent to the pathological locations, were included in the ROI. The defined region was filled with subsets of a stimulus arrangement with high spatial resolution (grid with 191 stimuli, see Fig. 1) to increase the density of test points within the ROI. The total number of test points did not exceed 160 . The individually specified locally condensed grids remained the same during the whole follow-up period in all patients.

Due to the increased number of test locations, the visual field examination was completed in two sub-sessions within a time window of $24 \mathrm{~h}$. The locally condensed grid was split into two complementary randomized subsets with an approximately equal number of test points to achieve reasonable examination durations for the two sessions. Nine identical stimulus locations were tested in both subgrids to control for intersession variability. This procedure was carried out according to a former publication [30] addressing this quality assurance.

\section{Patterns of progression}

Scotomata in baseline exams were defined as clusters of at least three contiguous visual field locations ((TD, $p<0.05$, with at least one $p<0.01)$ [27]). Any two visual field locations were considered as contiguous if they were neighbored in vertical, horizontal, or oblique direction within the $6^{\circ} \times 6^{\circ}$ distance. Progression was analyzed using trend analyses. A progressing visual field series was defined by the presence of a cluster of at least three contiguous, progressive, non-edge test points $[20,35]$ not crossing the horizontal midline [38] in two consecutive examinations in at least two of the same test locations according to the standards of the European Glaucoma Prevention Study [24]. Progressing visual fields series were assessed for the patterns in which their locations progressed relative to the initial scotomata. Progression of existing scotoma could occur in depth, size, or both depth and size. Local progression exclusively in depth was assumed if progressive test locations were contained within the initial scotoma cluster (red triangle symbol, Fig. 2). Exclusive local progression in size was assumed if progressive test locations occurred in locations (normal at baseline) adjacent to the initial scotoma cluster (red triangle and red frame symbol, Fig. 2). In addition to changes to existing scotomata, the occurrence of a new scotoma was assumed if there appeared a cluster of at least three pathological test points (TD, $p<0.05$ with at least one $p<0.01$ ) [27] within an initially normal area not adjacent to a previously identified scotoma.

The detection rate of progression in locally condensed grids was compared to the $6^{\circ} \times 6^{\circ}$ grid. The $95 \%$-confidence intervals (CI) were computed for the proportion of progressions that were detected with the help of the additional grid points. To determine whether the higher sensitivity of spatially condensed grids was bought at the expense of specificity, eyes were flagged if the session immediately after the first two consecutive sessions indicating progression did not reconfirm the confirmed progression. This criterion is

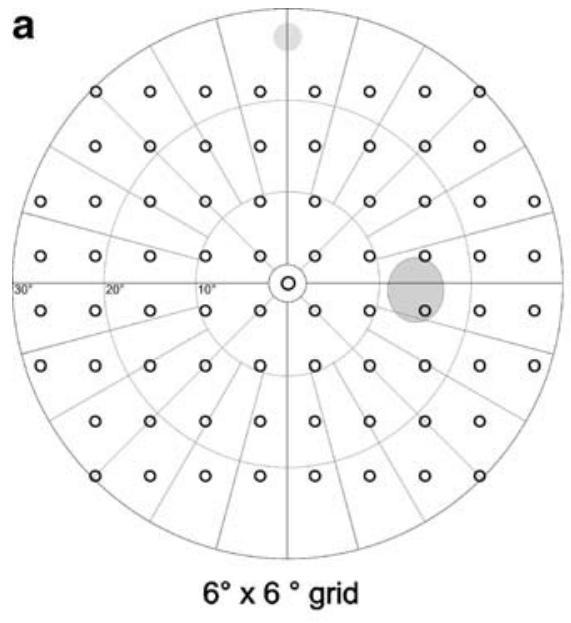

a

$6^{\circ} \times 6^{\circ}$ grid

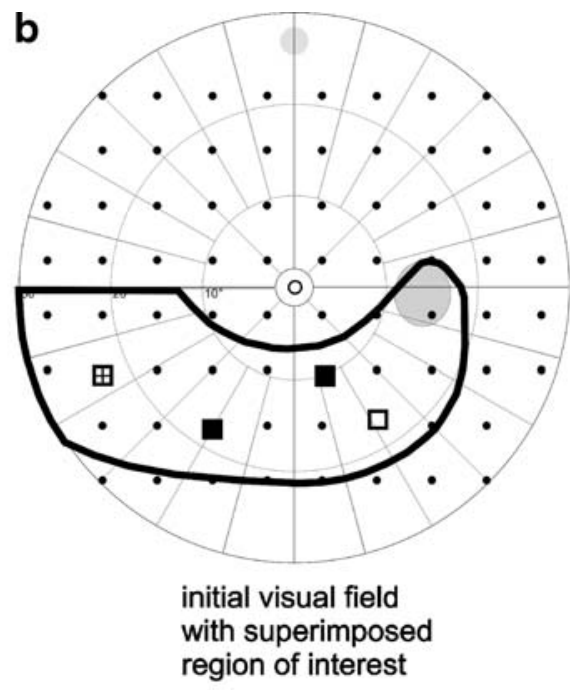

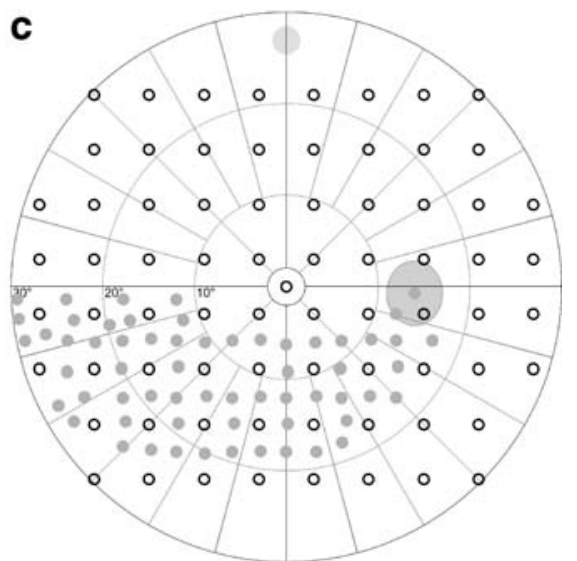

filled grey circles show the additional stimuli for the subsequent visual field examinations

Fig. 1 The $6 \times 6^{\circ}$ grid (a) was condensed in the region of interest (b) to achieve an individual locally condensed grid (c) 


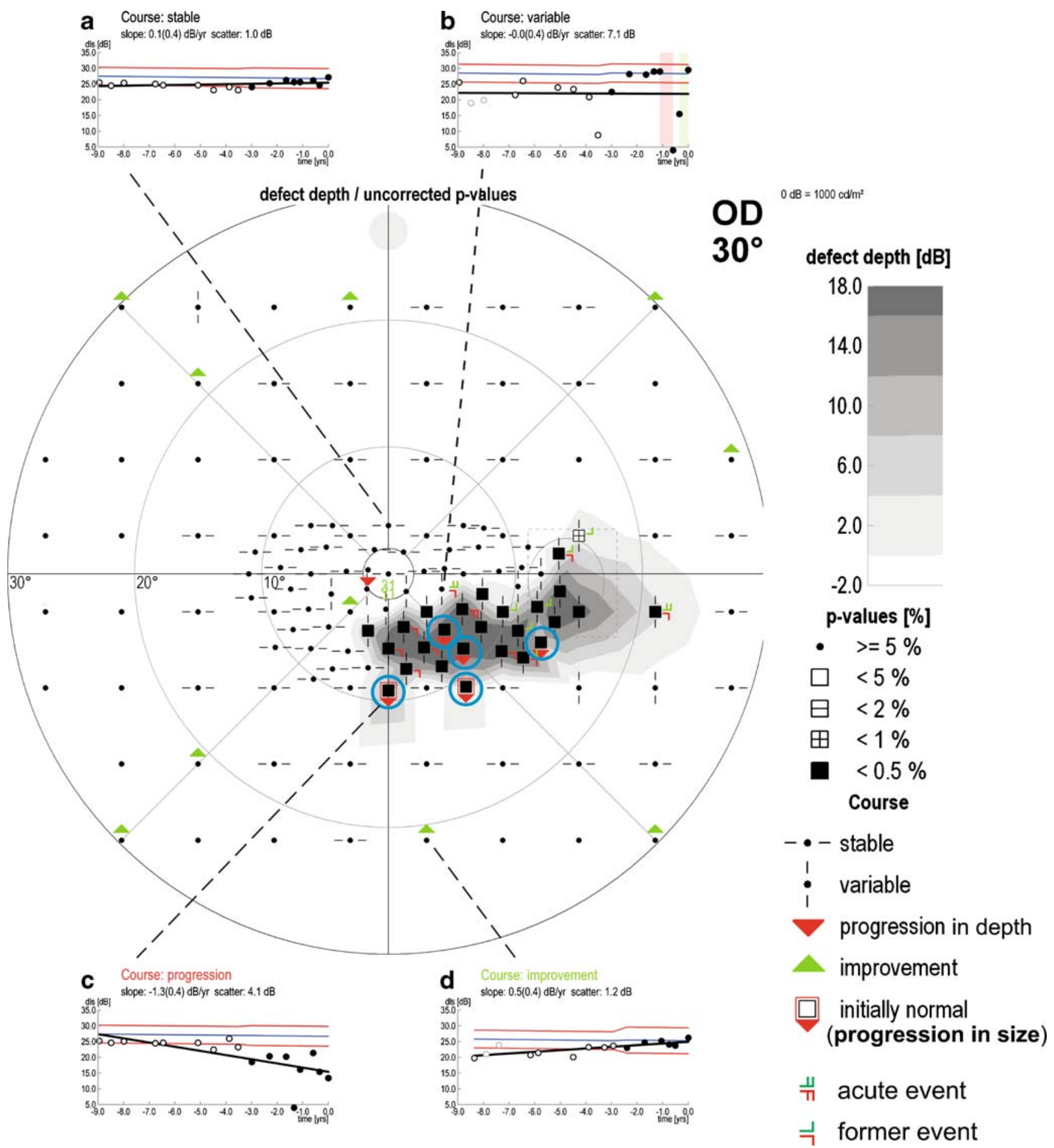

Fig. 2 Example of progression in depth and size (16 visits within 9 years). The test locations with confirmed progression are marked with red triangles. The initially normal and later pathologic test locations are marked with red frames. The four examples show the four different test point courses: (a) stable (b) variable (c) progression (d) improvement. The events are marked with red and green clasps in the test points and with red and green bars in the inset (b). The nearly horizontal blue lines show the age-related normative DLS values at the selected stimulus locations. The red lines show the 95\% CI. Blue circles highlight five progressive stimulus locations. Three of these non-edge locations are adjacent, i.e., they form a cluster with the distances less than or equal to the distances of the $6^{\circ} \times 6^{\circ}$ rectangular Humphrey grid. Therefore, this set of visual fields record is classified as progressive 
similar to that used in the final protocol of the Ocular Hypertension Treatment Study (OHTS) [21].

Progression trend analyses

To determine progression using trend analyses, in this study a modified pointwise linear regression (PLR) was applied for each test location [10]. It consisted of assuming one fixed variance for all eyes. That was necessary, as PLR was attempted with series that were too short to estimate variance as well as slope. Single locations were indicated as progressing if the slope of their regression was significantly below zero $(p<0.05)$, taking into account the magnitude of slope, the intersession time intervals, the number of sessions, and the time span of follow-up, and the scatter of DLS values over time: The shorter the follow-up and the fewer the examinations, the greater was the slope needed for the location to be flagged as progressing. Progression cut-offs using this regression technique are summarized in Table 1. For example, a slope exceeding $-1 \mathrm{~dB} /$ year when examining the visual field once a year for 5 years is flagged as progressing. Also, a slope exceeding $-1.6 \mathrm{~dB} /$ year is flagged as progressing if the subject had come in 6-month intervals for a 3 -year follow-up period.

The median time to detection of progression with spatially condensed grids was compared to that with the $6^{\circ} \times 6^{\circ}$ grid.

Event analyses

Event analyses were additionally employed to assess abrupt changes of local DLS at any test location during the follow-up sessions. At present, there is no generally accepted cut-off level for a progressive event, i.e., a critical magnitude of local test-retest DLS difference that warrants suspicion of glaucomatous progression. To obtain a cut-off, the frequency distribution of DLS test-retest differences for 86 suitable patients from the Tuebingen visual fields' database at all test locations between the third and fourth visual field examinations was assessed (Fig. 3) The distribution was found to be a mixture of two central Laplace-distributions. The lower 5\%quantile, $-10 \mathrm{~dB}$, of the more spread out distribution was set as criterion for a local perimetric event. The parameters of the Laplace-distributions were interpreted as expected absolute difference in stable and instable locations, and were estimated by maximum likelihood procedures.

Thus an event was defined as an abrupt local deterioration of DLS by more than $-10 \mathrm{~dB}$ between any two examinations (Figs. 2, 3). Additionally, clusters of events were evaluated (for definition of a "cluster", see above). Furthermore we assessed the events inside/outside a ROI and inside a cluster.

The study was carried out and evaluated according to the following five methodological standards set by Harper et al.
Table 1 Slope criteria for progressing test points depending on test interval and follow-up period

\begin{tabular}{|c|c|c|}
\hline Test interval & Follow-up period [years] & Slope $[\mathrm{dB} /$ year $]$ \\
\hline \multirow[t]{8}{*}{ Every 12 months } & 2.0 & -3.0 \\
\hline & 3.0 & -1.9 \\
\hline & 4.0 & -1.3 \\
\hline & 5.0 & -1.0 \\
\hline & 6.0 & -0.8 \\
\hline & 7.0 & -0.6 \\
\hline & 8.0 & -0.5 \\
\hline & 9.0 & -0.5 \\
\hline \multirow[t]{18}{*}{ Every 6 months } & 1.0 & -5.9 \\
\hline & 1.5 & -3.8 \\
\hline & 2.0 & -2.7 \\
\hline & 2.5 & -2.0 \\
\hline & 3.0 & -1.6 \\
\hline & 3.5 & -1.3 \\
\hline & 4.0 & -1.1 \\
\hline & 4.5 & -0.9 \\
\hline & 5.0 & -0.8 \\
\hline & 5.5 & -0.7 \\
\hline & 6.0 & -0.6 \\
\hline & 6.5 & -0.6 \\
\hline & 7.0 & -0.5 \\
\hline & 7.5 & -0.5 \\
\hline & 8.0 & -0.4 \\
\hline & 8.5 & -0.4 \\
\hline & 9.0 & -0.4 \\
\hline & 9.5 & -0.3 \\
\hline
\end{tabular}

(numbering according to Harper et al.) [12]: (1) specification of the spectrum composition, i.e., specification of the age, sex, initial disease stage, and the eligibility criteria; (2) avoidance of work-up (verification) bias, i.e., all patients were studied with both conventional and spatially condensed grids; (3) avoidance of review bias, i.e., both grids were analyzed independently; (4) presentation of precision of results for test accuracy for the most important results, i.e., 95\% CI is reported in comparison of detection of progression with conventional and condensed grids; and (5) reporting of indeterminate test results was not an issue since no indeterminate test results existed.

\section{Results}

Visual fields

We examined 260 visual fields of 41 eyes (four to 15 fields/ patient, median five fields/patient). Median follow-up 
Fig. 3 a Box-and-whiskers plot and histogram of 10,537 differences between individual local DLS thresholds at third and fourth examination. Small grey circles indicate values outside the inner fence. Vertical ticks on the whiskers mark the $0.5 \%$-, $2.5 \%-, 10 \%-, 90 \%-, 97.5 \%-$, and $99.5 \%$ quantiles. The smooth red line is the density of the mixture of $23.6 \%$ of a central Laplace-distribution with parameter 4.35 and $76.4 \%$ of a central Laplace-distribution with parameter 1.35. b Densities of the mixture (red encompassing) and its components (symmetric triangles) with a shaded area that represents $5 \%$ of the area under the solid symmetric triangle, when the density axis is not logarithmic. DLS differences in that area are considered events

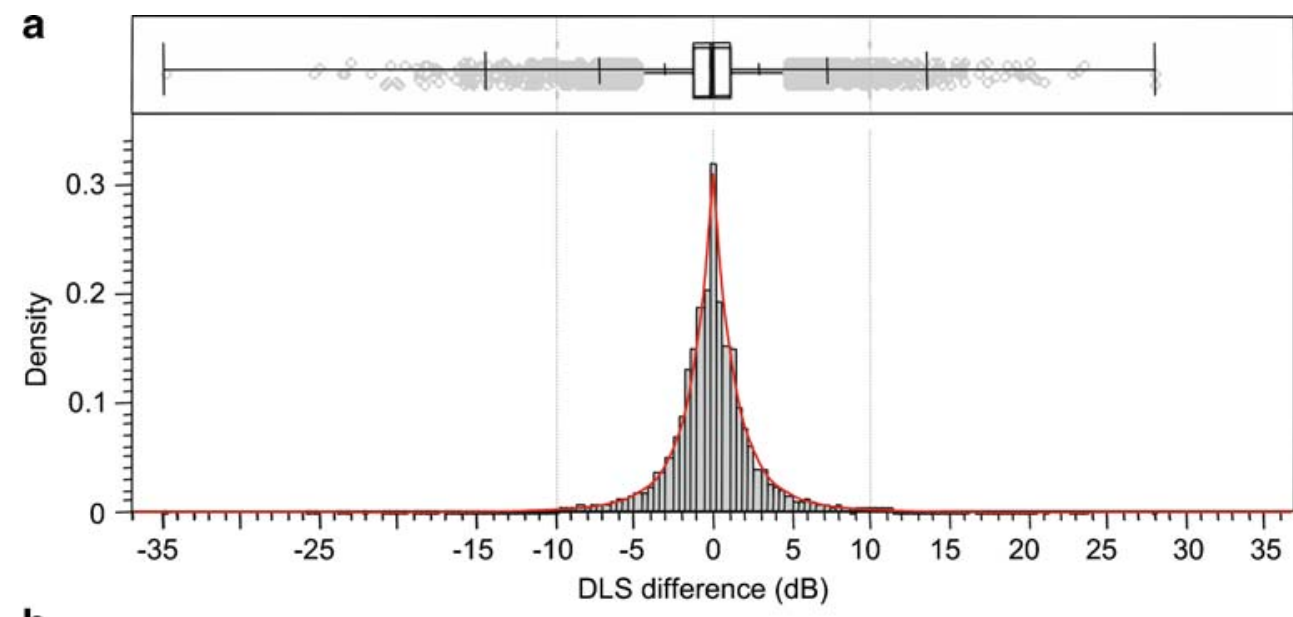

b

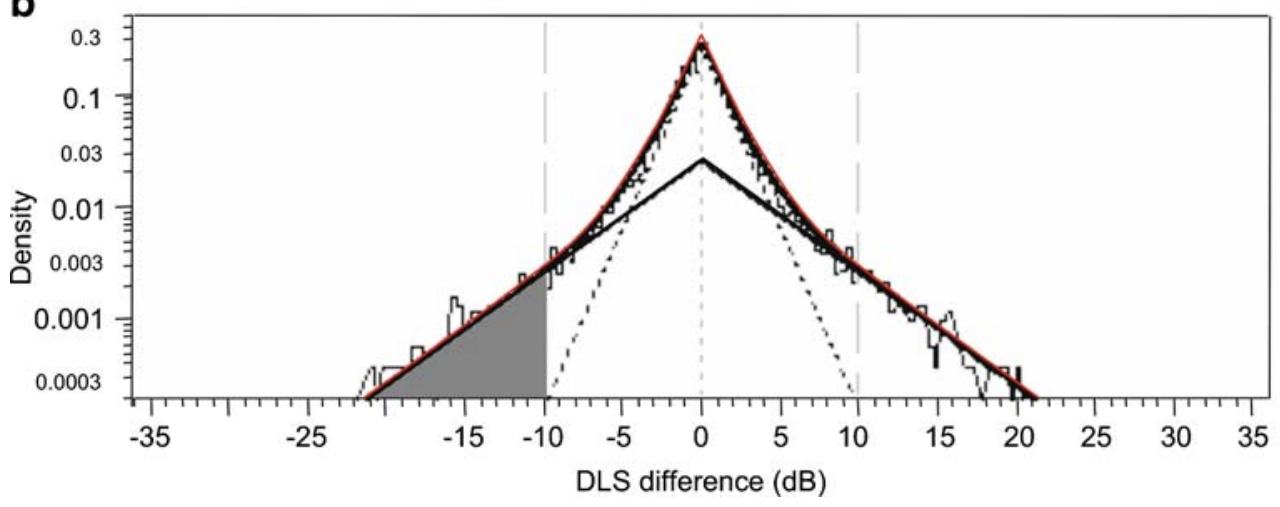

period was 2.5 years (range: 1.3 to 8.6 years) and median interval between the examinations was 6 months (range 229 months).

According to the Mills [27] glaucoma staging system, the scotoma had the following stages at the initial session: $22(54 \%)$ as early (stage 1), 11 eyes (27\%) as moderate (stage 2 ), five eyes (12\%) as advanced (stage 3), and three eyes $(7 \%)$ as severe (stage 4$)$. Mean deviation (MD) ranged from +1.6 to $-8.6 \mathrm{~dB}$ (mean $-1.8 \mathrm{~dB}$ ) and Loss Variance (LV) from 2.0 to $50.7 \mathrm{~dB}^{2}$ (mean $19.5 \mathrm{~dB}^{2}$ ) at the initial session. Mean change in $\mathrm{MD}$ during the follow-up in the progressive group was $+0.41 \mathrm{~dB}$ (range +4.3 to $-5.5 \mathrm{~dB}$ ).

Total duration of one visual field examination (subdivided into two sub-sessions) varied from $31.6 \mathrm{~min}$ to $52.6 \mathrm{~min}$ (median $41.5 \mathrm{~min}, 20.8 \mathrm{~min}$ per session) including a break of approximately $10 \mathrm{~min}$, which was not exactly recorded. We selected the subjects' third visit as representative for the examination times. Intersession variability ("Medium term fluctuation") turned out to be in the range of $1 \%$ of the total variation [30].

Patterns of progression

Confirmed progression of a visual field area with spatially condensed grid occurred in 11 (27\%) out of the 41 eyes. Of these eyes with visual field area progression, six of the 11
(55\%) revealed a combined progression in depth and size. In five eyes (45\%), there was deterioration exclusively in depth. There were no new scotomata or exclusive progression in size. Of the 30 eyes that did not meet our progression criterion, a progressive "cluster" was found in 16 eyes $(53 \%)$ whose presence was not confirmed in subsequent examinations. No progressive "clusters" could be found in visual field series of 14 eyes $(34 \%, 81$ visual field examinations).

We evaluated further the type of progression in the 11 eyes showing confirmed progression in a visual field area. There were altogether 198 progressive test locations: 183 (92\%) of them were located inside the ROIs, 49 (25\%) of them were initially normal, indicating progression in size, and $154(78 \%)$ of all progressive test locations were located within progressive clusters. Only one eye was flagged with a negative test occurring after two consecutive positive tests.

Progression trend analysis

Only four of the 11 progressive cases detected with spatially condensed grids would have been detected by applying the $6^{\circ} \times 6^{\circ}$ grid only (improvement by $7 / 11,95 \%$ CI 0.35 to 0.92 ). The delay from the detection of progression with condensed grids to that with the $6^{\circ} \times 6^{\circ}$ 
grid varied from 0 to 41 months or from 0 to 5 visits. Kaplan-Maier curves showing the time to detection of progression for both grids are shown in Fig. 4.

When all 11 progressions had been detected by SCOPE, at six visits or 32 months, just one progression would have been detected using the $6^{\circ} \times 6^{\circ}$ grid while it took ten visits or 73 months, until $4 / 11$ were detected using the $6^{\circ} \times 6^{\circ}$ grid (Fig. 5). When using Kaplan-Meier estimation, the median time gain through condensed grids was estimated to be six visits (lower limit of $95 \%$ CI two visits) or 41 months (lower limit of 95\% CI 19 months).

\section{Events}

Events were defined as a drop in DLS in a subsequent session. The event criterion was set to the $5 \%$-quantile of the distribution of instable locations, i.e., to $-10 \mathrm{~dB}$. The frequency distribution of 10,537 DLS test-retest differences for 86 suitable patients from the Tuebingen visual fields' database with 530 visual fields of the third and fourth examinations is shown in Fig. 3. The mean of absolute differences was estimated to be $1.35 \mathrm{~dB}$ in stable locations and $4.35 \mathrm{~dB}$ in instable locations.

Events by more than $-10 \mathrm{~dB}$ were seen in seven (64\%) of the 11 eyes showing progression evaluated with trend analyses. Events occurred in 57 test locations during the

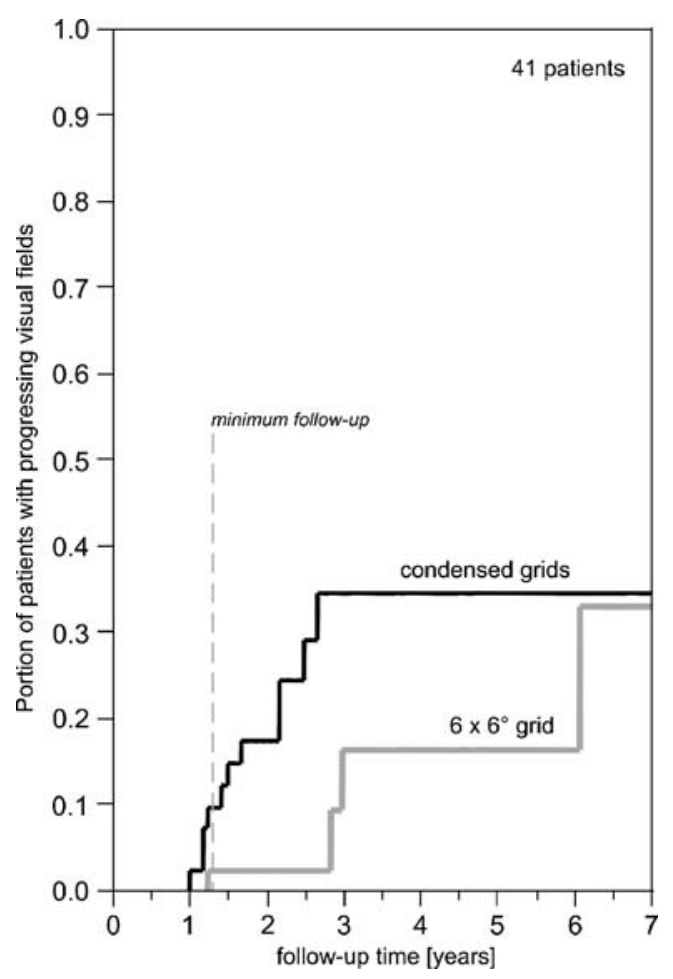

Fig. 4 Time for the first detection of progression for each grid. Locally condensed grids detect progression earlier than the $6^{\circ} \times 6^{\circ}$ grid. Minimum follow-up time was 16 months, mean follow-up time was 30 months ( 2.5 years $)$ follow-up period: $82 \%$ (47 of 57) of these events were located inside the ROIs, and 60\% (28 of 47) of these inside a progressive cluster. A cluster of three or more contiguous events that was confirmed within the subsequent 2 years appeared in five of the 11 progressing eyes. In eyes without trend progression we found ten events inside the ROIs, and no events outside the ROIs.

\section{Discussion}

Identifying progressive glaucomatous visual field defect remains a challenging task due to wide-ranging individual intra- and inter-test variability and due to the large number of different methods of progression analysis and different criteria for progression [10, 20, 23, 34]. Detection of progression at the earliest possible point in time in a sightthreatening disease is essential for an adequate onset and adjustment of therapeutic strategies. Delay between the clinical detection of nerve fiber atrophy and observing the defect in the visual field using conventional perimetry is up to 6 years [33]. In addition to using modern progression analyses techniques [29, 37], this delay can be shortened using stimulus arrangements with higher spatial resolution $[1,30,36,39]$. This is possible clinically, e.g., with the Octopus 101 instrument that allows for the generation of userdefined individual test point arrangements, referring to a smooth model of an age-corrected normal hill of vision, which is in contrast to the Humphrey Field Analyzer. Taking into account that glaucomatous visual field defects tend to progress in already damaged areas of the visual field $[4,25,28]$, it is reasonable to locally condense the grid in individually specified regions at increased risk of progression.

In a recent paper [30], we showed that locally condensed test grids significantly increase the detection rate of glaucomatous visual field loss in comparison to conventional $6^{\circ} \times 6^{\circ}$ grids. This study is intended to demonstrate that local grid condensation also enhances detection of progression of glaucomatous visual field loss.

The patterns of glaucomatous visual field progression found in this study did not differ remarkably from those identified with conventional grids in earlier studies despite of differences in study cohorts and progression criteria: The initial stage of glaucoma in Boden's [4] cohort was mild in about one-third and moderate in about two-thirds of cases, whereas in our cohort, about half had a mild glaucoma and half had moderate or severe glaucoma (corresponding to Mills IV) [27]. Mikelberg et al. [25] had included only progressive visual fields in their study. In Mikelberg's study there occurred also new scotomata, possibly due to longer follow-up.

In general, the expansion of the visual field defects conformed to the nerve fiber course in the retina. These findings agree with the results of Pascual et al. [28] and 

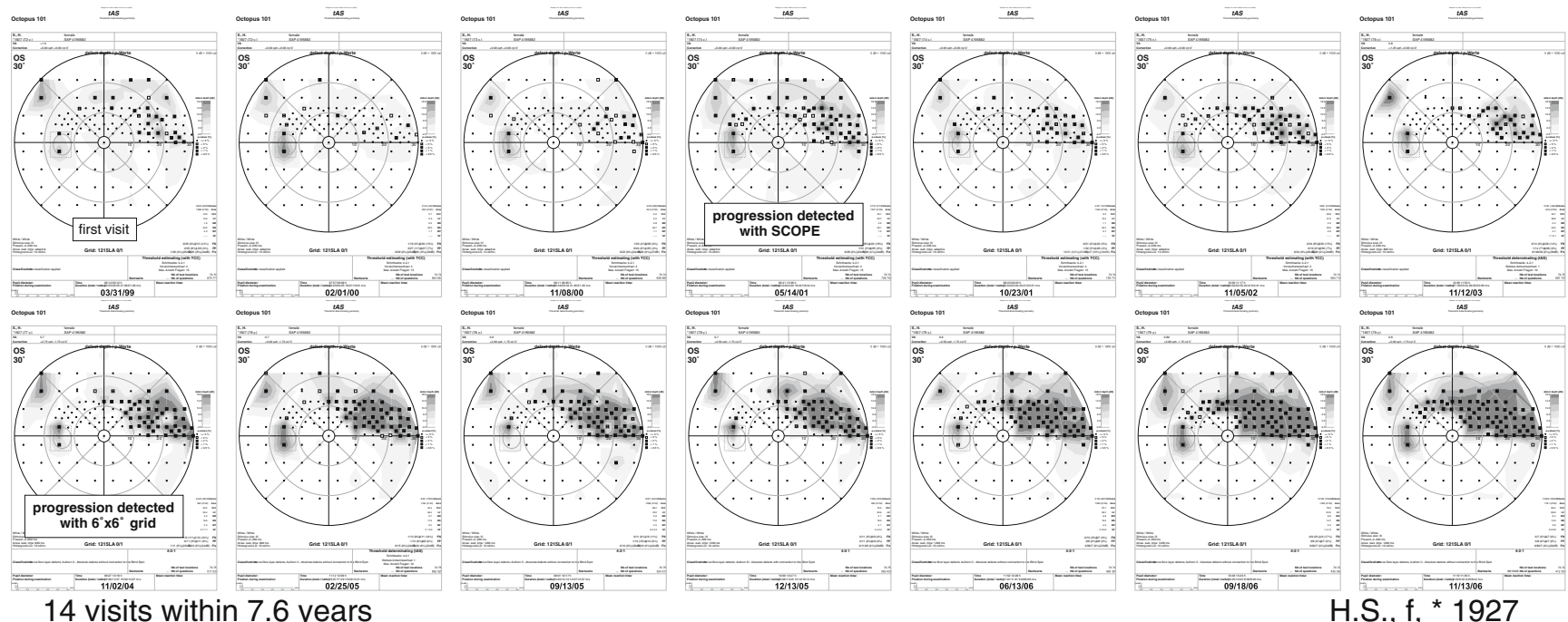

H.S., f, * 1927

Fig. 5 A representative course of progression of glaucomatous visual field defects, examined with additional test points in the visual field area adjacent to the existing scotomata (scotoma-oriented perimetry (SCOPE). SCOPE finds progression 3.5 years before the conventional $6^{\circ} \times 6^{\circ}$ grid

with structural progression in glaucoma [36]. Glaucoma is known to proceed slowly, and thus longer follow-up periods and larger study cohorts are needed to achieve more exact comparisons of the patterns of progression using different criteria for progression.

Despite a short follow-up, we could detect a considerable number of progressive visual fields in our current cohort. The progression rate of $27 \%$ after 3 years of followup was high compared to earlier reports: In the Early Manifest Glaucoma Trial (EMGT) [3], the progression rate was $69 \%$ during 8 years (median) of follow-up. The criterion used in this study to identify progressive visual fields called for a cluster of three progressive test locations. This is a commonly used criterion as published by Katz et al. [20]. The AGIS study uses this criterion as well [35]. This criterion can be made more stringent by requiring a cluster of four progressive test locations, which was used as criterion for a suspicion of visual field progression in the Canadian Glaucoma Study (CGS) [7]. That rate was 43.5\% after 8 years of follow-up in the CGS. Tightening the criterion would result in reducing the rate of progressive visual fields to $17 \%$ within 3 years in our cohort. However, when taking into account our finding that conventional grids find less than half of the progressive visual fields confirmed with spatially condensed grids, the progression rate of these earlier mentioned studies would presumably have been higher, if a grid with local test point condensation had been applied.

The appearance of clusters of abrupt "events" in more than one-third of the eyes showing progression by trend analysis suggests that such events may precede local progression, indicate a more rapid manifestation of glaucoma, or both. Observing (and when necessary responding to) "events" by increasing the frequency of visual field testing [18] or even intensifying the treatment of these patients could be worthwhile. In this study, an "event" was defined to be a sudden deterioration of more than $-10 \mathrm{~dB}$, a value that exceeds normal test-retest variability. Using a fixed criterion for all locations may not be appropriate since the test-retest variability is strongly dependent on the initial defect depth and the eccentricity of the test points under consideration [14]. However, any problems with using a fixed $\mathrm{dB}$ cut-off value should affect both the conventional and condensed grids equally since they contain similar locations and cover the same central $30^{\circ}$ of the visual field.

Most of the progressive test locations and events were situated inside the operator-defined ROI and were concentrated in the progressive clusters. Therefore, it is reasonable to assess potential progression in anatomically (e.g., optic nerve head or nerve fiber layer) or perimetrically (i.e., areas of earlier defects in the visual field) suspicious areas using spatially condensed test locations. However, applying locally condensed grids may not enhance the detection of diffusely progressive losses that do not have a scotomarelated clue for placement of the ROI.

Cataract formation can cause problems in determining progression of the scotomata, especially when using a TD plot instead of the pattern deviation plot that corrects for overall changes in visual field sensitivity. We decided to refer to the TD plot, since it has been shown to be more sensitive in detecting glaucomatous visual field progression [2]. The effect of cataract formation in the visual fields of glaucoma patients varies considerably between studies [5, $6,32]$. It has been shown that patients with mild or early scotomata tend to have a greater fall in the MD values due to cataract formation than patients with deeper scotomata 
[13]. Most of our patients had small scotoma areas as can be seen in the initial staging, and the mean change in the MD values during the follow-up in our cohort was very subtle, indicating that there should not be significant cataract formation effect the estimation of VFD progression. Finally, any effect of cataract would have influenced both the $6^{\circ} \times 6^{\circ}$ and condensed grids fairly equally. The slightly positive average change of MD may be caused by a general learning effect.

With every new diagnostic test, it is important to critically evaluate the test using generally accepted quality standards. Harper et al. [12] have examined the compliance with quality standards in ophthalmologic studies, and in general, only 2.9 of 7 evaluated quality standards were addressed. This study adheres to five of the seven standards set forth by Harper et al: (1) specification of the spectrum composition; (2) avoidance of work-up (verification) bias; (3) avoidance of review bias; (4) presentation of precision of results for test accuracy for the most important results, and (5) reporting of indeterminate test results, which was not an issue since no indeterminate test results existed. Two of Harper et al.'s quality standards were not fulfilled: (6) subgroups were not analyzed separately because of the small sample size and (7) reproducibility of test results was not presented separately since, by definition, progression was based on reproducibility of the test. Harper et al. also recommended three additional standards: (1) definition of the gold standard, (2) independence of the gold standard, i.e., the test under evaluation should not be performed as part of the gold standard, and (3) appropriateness of the study population, i.e., avoidance of selection bias. We did not evaluate the gold standard separately (additional standard 2), as the condensed test locations were added to the test locations of the gold standard.

Variability in automated static perimetry in glaucomatous visual field loss can cause mis-interpretation of progression of glaucoma [14, 15]. Addition of test locations may result in both, false-stable and false-progressive results. The evaluation was focused on the analysis of potential falseprogressive rates, as this condition would result in additional diagnostic and therapeutic activities, inducing further costs and also potentially further uncertainty and discomfort of the patient(s). Thus, confirmation of visual field loss is essential to avoid false-positive progression and overtreatment [22]. We could evaluate potential false progressive results similarly to methods used in earlier reports [21] that required at least two consecutive visual fields to indicate progression, but did not have enough follow-up tests to increase the criterion to the repeatability in three consecutive visual fields.

Local test point condensation had better stability compared to conventional grids. Only three eyes that had confirmed progression by local test point condensation demonstrated a non-progressive visual field at some time after confirming progression. Using the final criterion of the OHTS study [21], locally condensed grids detected progression in three consecutive examinations in our cohort in $73 \%$ of eyes demonstrating progression at some time during follow-up. Furthermore, after the first confirmation of progression, locally condensed grids detected progression in $91 \%$ of all the following 54 consecutive examinations. Thirtyseven percent of the eyes in our cohort remained stable in all 85 visual field examinations. This repeatability suggests that the increased number of progressive findings is not due to an increase in false-positive results. However, as most patients in our cohort were examined only four to six times, the possibility of randomness still exists. Thus we cannot rule out a lower specificity of the locally condensed grid approach completely. This matter requires further research.

Due to the change in our perimetric instruments (the TCC was replaced with the Octopus 101), the perimeter changed in the course of our study in six progressive eyes, but the test grid was preserved across devices accounting for the flat screen of the TCC and the cupola of the Octopus 101 perimeter. Progression was already detected in five eyes before the change of the instrument, and the Octopus 101 confirmed the progression. Progression was detected after the change to the Octopus 101 in only one eye. In this case, the condensed grid and the progressive cluster were located inside $15^{\circ}$ of eccentricity, where the risk of spatial distortion of the grids between the instruments is negligible. It has been shown that there are only minor differences in DLS values between the TCC and the Octopus 101 [16], so the replacement of the TCC with the Octopus 101 should not have affected the further visual field findings of these six progressive eyes tested on both devices.

For this study, the examination had to be split into two sub-sessions to reduce the test durations to reasonable times. The development of a faster strategy (German adaptive Threshold Estimation, GATE) [31] to considerably reduce the examination time makes it possible to apply locally condensed stimulus arrangements within one session.

Furthermore, test point density can be reduced in "regions of no interest", i.e., widespread areas of absolute defects or areas with repeated confirmed normal DLS values.

It is important to consider the benefit of the increased effort due to additional test time. Using the same progression analysis technique and examination sequence, condensed test grids detected progression on average of 6 visits (41 months) earlier than conventional $6^{\circ} \times 6^{\circ}$ grid. Taking the irreversible nature of glaucomatous optic neuropathy into account, this is a considerable benefit.

Theoretically, increased effort to detect the visual fields' change could be invested in (a) enhanced spatial resolution, as is done in this study by local condensation of the test grid; (b) improving the precision of the threshold estimation of a 
standard grid; or (c) increasing the frequency of examinations with the standard grid. The concept of the present study is not adequate to address this question. However, considering the inherent variability of local threshold estimates, often exceeding $+3 \mathrm{~dB}$ (that means half or double of the original luminance level) it seems to be very unlikely that clause (b) or (c) are a very successful option. On the other hand, a spontaneous inter-session fluctuation of scotoma size is much smaller than fluctuation of scotoma depth. Therefore, focusing effort on enhanced spatial resolution as done in this study, is the logical consequence.

An abrupt change of DLS by more than $-10 \mathrm{~dB}$ (perimetric event) occurred in more than $50 \%$ of eyes showing progression in our cohort. A comparison with the literature is difficult, especially due to differing event criteria. A step size of $10 \mathrm{~dB}$ as applied in this study is the greatest (most conservative) value compared to other studies [2, 11, 34].

Due to a lack of real-time tracking in conventional automated perimetry, there is an inherent limitation of spatial test point condensation: patient-related fixation instability results in deviations from the fixation target by a minimum of $1-2^{\circ}$ [8]. Therefore, an additional test point below this critical distance would introduce an "artificial noise" to the perimetric results and scotoma edges. Future microperimetric techniques applying real-time fundus tracking should be able to overcome this shortcoming. The development of a standardized automated algorithm for test point condensation (autoSCOPE) is underway.

In conclusion, individually determined locally condensed grids enable the detection of a glaucomatous visual field progression more frequently and also earlier than conventional grids at the expense of an increased examination time. The most common pattern of visual field progression in glaucoma is a combined deepening and expansion of an existing scotoma.

Acknowledgements This research was supported in part by Finland's eye foundation, Evald and Hilda Nissi Foundation and Finnish Ophthalmologists council.

Open Access This article is distributed under the terms of the Creative Commons Attribution Noncommercial License which permits any noncommercial use, distribution, and reproduction in any medium, provided the original author(s) and source are credited.

\section{References}

1. Airaksinen PJ, Heijl A (1983) Visual field and retinal nerve fibre layer in early glaucoma after optic disc haemorrhage. Acta Ophthalmol Copenh 61:186-194

2. Artes PH, Nicolela MT, LeBlanc RP, Chauhan BC (2005) Visual field progression in glaucoma: total versus pattern deviation analyses. Invest Ophthalmol Vis Sci 46:4600-4606
3. Bengtsson B, Leske MC, Hyman L, Heijl A (2007) Fluctuation of intraocular pressure and glaucoma progression in the early manifest glaucoma trial. Ophthalmology 114:205-209

4. Boden C, Blumenthal EZ, Pascual JP, McEwan G, Weinreb RN, Medeiros F, Sample PA (2004) Patterns of glaucomatous visual field progression identified by three progression criteria. Am J Ophthalmol 138:1029-1036

5. Budenz DL, Feuer WJ, Anderson DR (1993) The effect of simulated cataract on the glaucomatous visual field. Ophthalmology 100:511-517

6. Carrillo MM, Artes PH, Nicolela MT, LeBlanc RP, Chauhan BC (2005) Effect of cataract extraction on the visual fields of patients with glaucoma. Arch Ophthalmol 123:929-932

7. Chauhan BC, LeBlanc RP, Nicolela MT (2006) Canadian Glaucoma Study: 1. Study design, baseline characteristics, and preliminary analyses. Can J Ophthalmol 41:566-575

8. Eizenman M, Trope GE, Fortinsky M, Murphy PH (1992) Stability of fixation in healthy subjects during automated perimetry. Can J Ophthalmol 27:336-340

9. European Glaucoma Society (2003) Terminology and guidelines for glaucoma, 2nd edn. Editrice Dogma, Savona

10. Gardiner SK, Crabb DP (2002) Examination of different pointwise linear regression methods for determining visual field progression. Invest Ophthalmol Vis Sci 43:1400-1407

11. Garway-Heath D (2006) Event- and trend-based visual field analyses in clinical trails of glaucoma neuroprotection. In: Weinreb RN (ed) Glaucoma neuroprotection. Wolters Kluwer Health Inc, Philadelphia, pp 83-94

12. Harper R, Reeves B (1999) Compliance with methodological standards when evaluating ophthalmic diagnostic tests. Invest Ophthalmol Vis Sci 40:1650-1657

13. Hayashi K, Hayashi H, Nakao F, Hayashi F (2001) Influence of cataract surgery on automated perimetry in patients with glaucoma. Am J Ophthalmol 132:41-46

14. Heijl A, Lindgren A, Lindgren G (1989) Test-retest variability in glaucomatous visual fields. Am J Ophthalmol 108:130-135

15. Henson DB, Darling MN (1995) Detecting progressive visual field loss. Ophthalmic Physiol Opt 15:387-390

16. Hermann A, Paetzold J, Vonthein R, Krapp E, Rauscher S, Schiefer U (2005) Conversion of age-specific normative differential luminance sensitivity obtained with the Tuebingen Computer Campimeter and the Octopus 101 perimeter. Invest Ophthalmol Vis Sci 46:E-Abstract 4321

17. Hermann A, Paetzold J, Vonthein R, Krapp E, Rauscher S, Schiefer U (2008) Age-dependent normative values for differential luminance sensitivity in automated static perimetry using the Octopus 101. Acta Ophthalmol 86:446-455

18. Jansonius NM (2006) Towards an optimal perimetric strategy for progression detection in glaucoma: from fixed-space to adaptive inter-test intervals. Graefes Arch Clin Exp Ophthalmol 244: 390-393

19. Jonas JB, Gusek GC, Naumann GO (1988) Optic disc morphometry in chronic primary open-angle glaucoma. I. Morphometric intrapapillary characteristics. Graefes Arch Clin Exp Ophthalmol 226:522-530

20. Katz J, Sommer A, Gaasterland DE, Anderson DR (1991) Comparison of analytic algorithms for detecting glaucomatous visual field loss. Arch Ophthalmol 109:1684-1689

21. Keltner JL, Johnson CA, Levine RA, Fan J, Cello KE, Kass MA, Gordon MO (2005) Normal visual field test results following glaucomatous visual field end points in the Ocular Hypertension Treatment Study. Arch Ophthalmol 123:1201-1206

22. Keltner JL, Johnson CA, Spurr JO, Kass MA, Gordon MO, Ocular hypertension treatment study group (OHTS) (2000) Confirmation of visual field abnormalities in the ocular hypertension treatment study. Arch Ophthalmol 118:1187-1194 
23. Lee AC, Sample PA, Blumenthal EZ, Berry C, Zangwill L, Weinreb RN (2002) Infrequent confirmation of visual field progression. Ophthalmology 109:1059-1065

24. Miglior S, Zeyen T, Pfeiffer N, Cunha-Vaz J (2002) The European glaucoma prevention study design and baseline description of the participants. Ophthalmology 109:1612-1621

25. Mikelberg FS, Drance SM (1984) The mode of progression of visual field defects in glaucoma. Am J Ophthalmol 98:443-445

26. Mikelberg FS, Schulzer M, Drance SM, Lau W (1986) The rate of progression of scotomas in glaucoma. Am J Ophthalmol $101: 1-6$

27. Mills RP, Budenz DL, Lee PP, Noecker RJ, Walt JG, Siegartel LR, Evans SJ, Doyle JJ (2006) Categorizing the stage of glaucoma from pre-diagnosis to end-stage disease. Am J Ophthalmol 141:24-30

28. Pascual JP, Schiefer U, Paetzold J, Zangwill LM, Tavares IM, Weinreb RN, Sample PA (2007) Spatial characteristics of visual field progression determined by Monte Carlo simulation: diagnostic innovations in glaucoma study. Invest Ophthalmol Vis Sci 48:1642-1650

29. Sample PA, Boden C, Zhang Z, Pascual JP, Lee TW, Zangwill LM, Weinreb RN, Crowston JG, Hoffmann EM, Medeiros FA, Sejnowski T, Goldbaum M (2005) Unsupervised machine learning with independent component analysis to identify areas of progression in glaucomatous visual fields. Invest Ophthalmol Vis Sci 46:3684-3692

30. Schiefer U, Flad M, Stumpp F, Malsam A, Paetzold J, Vonthein R, Denk PO, Sample PA (2003) Increased detection rate of glaucomatous visual field damage with locally condensed grids: a comparison between fundus-oriented perimetry and conventional visual field examination. Arch Ophthalmol 121:458-465
31. Schiefer U, Pascual JP, Edmunds B, Feudner E, Hoffmann EM, Johnson CA, Lagrèze W, Pfeiffer N, Sample PA, Staubach F, Weleber RG, Vonthein R, Krapp E, Paetzold J (2009) Comparison of the new perimetric "German Adaptive Threshold Estimation" (GATE) strategy with conventional full-threshold and SITA Standard strategies. Invest Ophthalmol Vis Sci 50:488-494

32. Smith SD, Katz J, Quigley HA (1997) Effect of cataract extraction on the results of automated perimetry in glaucoma. Arch Ophthalmol 115:1515-1519

33. Sommer A, Katz J, Quigley HA, Miller NR, Robin AL, Richter RC, Witt KA (1991) Clinically detectable nerve fiber atrophy precedes the onset of glaucomatous field loss. Arch Ophthalmol 109:77-83

34. Spry PG, Johnson CA (2002) Identification of progressive glaucomatous visual field loss. Surv Ophthalmol 47:158-173

35. The Advanced Glaucoma Intervention Study Investigators (1994) Advanced glaucoma intervention study. 2. Visual field test scoring and reliability. Ophthalmology 101:1445-1455

36. Tuulonen A, Lehtola J, Airaksinen PJ (1993) Nerve fiber layer defects with normal visual fields. Do normal optic disc and normal visual field indicate absence of glaucomatous abnormality? Ophthalmology 100:587-598

37. Viswanathan AC, Fitzke FW, Hitchings RA (1997) Early detection of visual field progression in glaucoma: a comparison of PROGRESSOR and STATPAC 2. Br J Ophthalmol 81:1037-1042

38. Vonthein R, Paetzold J, Stumpp F, Flad M, Schiefer U (2004) Criteria for progression of glaucomatous visual field loss considering slope and scatter of local serial differential luminance sensitivity data. Invest Ophthalmol Vis Sci 45:E-Abstract 235

39. Westcott MC, McNaught AI, Crabb DP, Fitzke FW, Hitchings RA (1997) High spatial resolution automated perimetry in glaucoma. Br J Ophthalmol 81:452-459 